\title{
Finitely Generated Modules's Uniserial Dimensions Over a Discrete Valuation Domain
}

\author{
Samsul Arifin ${ }^{1,2, *}$, Hanni Garminia ${ }^{1}$, Pudji Astuti $^{1}$ \\ ${ }^{1}$ Algebra Research Group, Faculty of Mathematics and Natural Sciences, Institut Teknologi Bandung, Jawa Barat, 40132, Indonesia \\ ${ }^{2}$ Statistics Department, School of Computer Science, Bina Nusantara University, Jakarta, 11480, Indonesia
}

Received April 20, 2021; Revised June 10, 2021; Accepted June 25, 2021

Cite This Paper in the following Citation Styles

(a): [1] Samsul Arifin, Hanni Garminia, Pudji Astuti, "Finitely Generated Modules's Uniserial Dimensions Over a Discrete Valuation Domain," Mathematics and Statistics, Vol.9, No.4, pp. 521-526, 2021. DOI: 10.13189/ms.2021.090411

(b): Samsul Arifin, Hanni Garminia, Pudji Astuti, (2021). Finitely Generated Modules's Uniserial Dimensions Over a Discrete Valuation Domain. Mathematics and Statistics, 9(4), 521-526. DOI: 10.13189/ms.2021.090411

Copyright @2021 by authors, all rights reserved. Authors agree that this article remains permanently open access under the terms of the Creative Commons Attribution License 4.0 International License

\begin{abstract}
We present some methods for calculating the module's uniserial dimension that finitely g enerated o ver a DVD in this article. The idea of a module's uniserial dimension over a commutative ring, which defines how far the module deviates from being uniserial, was recently proposed by Nazemian etc. They show that if $R$ is Noetherian commutative ring, which implies that every finitely generated module over $\mathrm{R}$ has uniserial dimension. Ghorbani and Nazemians have shown that $\mathrm{R}$ is Noetherian (resp. Artinian) ring if only the ring $\mathrm{R} \mathrm{X} \mathrm{R}$ has (resp. finite) valuation dimension. The finitely generated modules over valuation domain are further examined from here. However, since the region remains too broad, further research into the module's uniserial dimensions that finitely generated over a DVD is needed. In the case of a DVD $\mathrm{R}$, a finitely generated module over $\mathrm{R}$ can, as is wellknown, be divided into a direct sum of torsion and a free module. Therefore, first, we present methods for determining the primary module's uniserial dimension, and then followed by methods for the general finitely generated module. As can be observed, the module's uniserial dimension is a function of the elementary divisors and the rank of the non torsion module item, which is the major finding of this work.
\end{abstract}

Keywords Uniserial Dimension, Valuation Dimension, Finitely Generated, DVD

\section{Introduction}

Uniserial modules are valuation rings that have been generalized. It is common knowledge that a set of all ideals ordered by inclusion can be described as a valuation ring. The concept of a valuation ring investigated in [9], [10], [13], and [15].On the other hand, uniserial modules can be classified as an assembly of all submodules that have been entirely arranged. The characteristics of the [8], [7] and [22] uniserial module are examining. The uniserials dimension of a module is a measure of how much it deviates from its uniserial state. Nazemian [17] was the first to present the concept of this uniserial dimension.

Some properties of uniserial-dimension modules have been investigated. For every $R$ and ordinal number $\alpha$, Nazemian [17] showed that over $\mathrm{R}$, there is a module with the uniserial dimension $\alpha$. In addition, if and only if a commutative ring $\mathrm{R}$ is Noetherian any finitely produced module over $\mathrm{R}$ has uniserials dimension. A finitely produced R-modules has a (resp. finite) uniserial dimension only if it has a Noetherian (resp. Artinian) commutative ring $\mathrm{R}$. Any $\mathrm{M}$ factor module is uniserial when $\mathrm{R}$-module $\mathrm{M}$ is uniserial. In the literature, she also showed that all module of Noetherian are uniserial. Every Artinian module, like every Noetherian module, thus has its finite uniserial dimension.

It should be noted that a finitely produced module $M$ begins to decompose over a PID R with the first step:

$$
M=M_{\text {free }} \oplus M_{\text {tor }}
$$

where $M_{\text {free }}$ represents a free module and $M_{\text {tor }}$ represents a torsion module, each of which is finitely generated over $R$. Since any discrete valuation domain is PID, so we can also use the same methods for a finitely produced module's decomposition over a DVD. Next, we'll look at the decomposition of the torsion side, $M_{t o r}$, which involves splitting $M_{t o r}$ into direct sums of primary's submodules. In addition, a direct sum of cyclic submodules could be constituted of the primary module, with the orders of the cyclic generators being referred to as the module's elementary divisors (see Roman in [19]). There- 
fore, we can get the uniserial dimension of this torsion module. On the other hand, in addition, we also focus on the decomposition of the free part, $M_{f r e e}$, over a DVD with finite rank, i.e. $M_{\text {free }}$ can be broken down into a direct sum of finite free and cyclic's submodules. Hence, we also can determine the uniserial dimension of this free module $M_{\text {free }}$ part.

The foregoing facts raise the question about how to decide the uniserial dimension of a finitily generated module $M$ over a DVD $R$, where $M=M_{\text {free }} \oplus M_{\text {tor }}$, which is the topic of this paper. In Section 2, we provide a technique for calculating the torsion module, Mtor, on DVD, uniserial dimensions of the module and also demonstrate that the elementary divisor function of the module. The method to determine a finitely produced free module uniserial dimension, $M_{\text {free }}$, with finite rank over a DVD is given in Section 3, as our second main result. We close this paper with a conclusion in Section 4.

\section{Finitely Generated Primary Mod- ules's Uniserial Dimension}

We offer a technique in this part to determine the uniserial size of a primary module finitely produced over a DVD. The uniserial dimension of finitely created primary modules is more learned in [3]. First of all imagine that $R$ is a $p$ prime element DVD and $m$ is a module over $R$ finely produced. First, we look at some uniserial modules, which are connected to ordinal numbers and created through transfinite induction (see [21]). It is important to capture or give the precise definition of an uniserial module. In Nazemian [17], we define for any ordinal number $\alpha \geq 1$ and $R$-module $M$ :

1. $\zeta_{1}=\{M \mid M$ uniserial $\}$

2. $\zeta_{\alpha}=$

$\left\{M \mid(\forall N<M)\left(M / N \nsubseteq M \Longrightarrow M / N \in \underset{\beta<\alpha}{\cup} \zeta_{\beta}\right)\right\}$

Note that the following definitions is very important.

Definition 2.1. Nazemian. [17]. Assume that $R$ is a ring, $M$ is the $R$-modules.

1. The smallest ordinal number $\alpha$, is said uniserial dimension from the module $M$, denoted by u.s.dim $(M)=\alpha$, if $M \in \zeta_{\alpha}$.

2. u.s.dim $(M)=0$ if $M=0$.

3. We mentioned that " $M$ has no uniserial dimension", $\forall \alpha \geq$ 1 with $M \neq 0$ but $M \notin \zeta_{\alpha}$.

The following example demonstrates the concept of uniserial dimension in $R$-modules.

Example 2.2. Let $M=\mathbb{Z}_{7} \oplus \mathbb{Z}_{7}$ be a $\mathbb{Z}$-module, it can be demonstrated that u.s.dim $(M)=2$. Now we've got:

$$
M=\mathbb{Z}_{7} \oplus \mathbb{Z}_{7}=\{(0,0),(0,1), \ldots,(6,6)\}
$$

with order 49. We have $M / N \in \zeta_{1}$ for any $N$ submodules of $M$, therefore $M \in \zeta_{2}$ is the result. Submodules of $M$, on the other hand, do exist:

$$
\begin{aligned}
& -N_{1}=\{(0,0),(0,1),(0,2),(0,3),(0,4),(0,5),(0,6)\} ; \\
& \text { - } N_{2}=\{(0,0),(1,2),(2,4),(3,6),(4,1),(5,3),(6,5)\} ;
\end{aligned}
$$

Therefore $N_{1} \nsubseteq N_{2}$ and $N_{1} \nsupseteq N_{2}$, implying that $M$ isn't uniserial, resulting in $M \notin \zeta_{1}$. As a result, u.s.dim $(M)=2$ is implied.

Certain uniserial modular characteristics have been found (see [17]). Moreover, the uniserial dimension of the regular modules was shown by [2] through a PID. We would also want information about decomposing a finished module through the PID. Remember that, if $p \in R$ is a primary element, a $p$ primary module has a $p$ order. In [19], the following traits were found: A finitely generrated module $M$ is the direct sums of a finitely generrated, free and torsion's $R$-modules over a PID $R$ :

$$
M=M_{\text {free }} \oplus M_{\text {tor }} .
$$

The torsion of the Mtor part is unique because the torsion element must be composed of $M$ and $M_{\text {free }}$ only part to isomorphism, i.e. free part rank. If $M$ is the major torsion module finished with an order of $p^{e}$ over a PID $R$, thus $M$ become a direct sums:

$$
M=\left\langle x_{1}\right\rangle \oplus \ldots \oplus\left\langle x_{k}\right\rangle
$$

of cyclic's submodules along with anihilators ann $\left\langle x_{i}\right\rangle=\left\langle p^{e_{i}}\right\rangle$ that may be arranged in ascending order:

$$
\text { ann }\left\langle x_{1}\right\rangle \subseteq \text { ann }\left\langle x_{2}\right\rangle \subseteq \ldots \subseteq \text { ann }\left\langle x_{k}\right\rangle,
$$

or equivalently,

$$
e=e_{1} \geq e_{2} \geq \ldots \geq e_{k}
$$

Further, the elementary divisors of $M$ are referred to as $p^{e_{i}}$ where $i=1,2, \ldots, k$ (see [6] and [20]).

Lemma 2.3. Assume $M$ become a finitely generated primary $R$-module with a cyclic decomposition

$$
M=\left\langle x_{1}\right\rangle \oplus\left\langle x_{2}\right\rangle \oplus \cdots \oplus\left\langle x_{k}\right\rangle
$$

where the order of $x_{i} o\left(x_{i}\right)=p^{e_{i}}, i=1,2, \ldots, k$ for some

$$
e=e_{1} \geq e_{2} \geq \cdots \geq e_{k} \geq 1 \text {. }
$$

Let $x \in M x \neq 0$ such that $p x=0$. Thus, $\exists j \in\{1, \ldots, k\} \ni$

$$
M /\langle x\rangle \cong\left\langle y_{1}\right\rangle \oplus\left\langle y_{2}\right\rangle \oplus \cdots \oplus\left\langle y_{k}\right\rangle
$$

for some $y_{i}, i=1,2, \ldots, k$ with properties

$$
o\left(y_{i}\right)= \begin{cases}p^{e_{i}-1} & \text { if } i=j \\ p^{e_{i}} & \text { if } i \neq j\end{cases}
$$


Note: $o(y)=1$ is equivalent to $y=0$.

The following theorem now exists, i.e. a technique to verify the uniserial dimension of a finished primary module across a DVD created with the element $k$ in all order of $p$ as well as the primary module in a uniserial dimension over a DVD which produced using $k$ s element in the order $p^{l i}$ in which $i=1, \ldots, k$ is created.

Theorem 2.4. Arifin [3]. Supposse $M$ become a finitely generrated primary $R$-module along a cyclic decomposition

$$
M=\left\langle x_{1}\right\rangle \oplus\left\langle x_{2}\right\rangle \oplus \cdots \oplus\left\langle x_{k}\right\rangle
$$

where the order of $x_{i} o\left(x_{i}\right)=p^{e_{i}}, i=1,2, \ldots, k$ for some descending finite sequence of integers

$$
e=e_{1} \geq e_{2} \geq \cdots \geq e_{k} \geq 1
$$

and $k \geq 2$. Then u.s.dim $(M)=\sum_{i=1}^{k} e_{i}$.

\section{Finitely Generatd Modules's Unise- rial Dimension}

After studying the uniserial dimensions of the torsion module part $M_{\text {tor }}$ (see Theorem 2.4, the next discussion is about the uniserial dimension for the free module part $M_{\text {free }}$.Main conclusions of this part include the 3.8 theory that is a way of deducting a finitely produced free modul's uniserial dimension across a finite rank discrete valuation domain.First, the underlying lemma is necessary.

Lemma 3.1. Assume $M$ become a free R-module of rank $n \geq$ 2 and $x \in M, x \neq 0$. Then $M /\langle x\rangle \cong R^{n-1}$ or $M /\langle x\rangle \cong$ $R^{n-1} \oplus\langle y\rangle$ for a torsion element $y$ with order $o(y)=p^{k}$ for certain positive integer $k$.

Proof. We have $\langle x\rangle$ is a submodule of $M$ with rank one. According to Theorem 6.7 [19, p.145] there exists $\left\{x_{1}, x_{2}, \ldots, x_{n}\right\}$ a basis of $M$ and $r \in R, r \neq 0$ such that $\left\{r x_{1}\right\}$ is a basis of $\langle x\rangle$. If $r$ is unit then we have $R^{n} /\langle x\rangle \cong$ $R^{n-1}$.

Suppose $r$ is not unit and $r=p^{k} u$ for some $u \in R$ unit and $k$ positive integer. We have

$$
M /\langle x\rangle=\left(\oplus_{i=1}^{n}\left\langle x_{i}\right\rangle\right) /\left\langle u p^{k} x_{1}\right\rangle=\oplus_{i=1}^{n}\left\langle\bar{x}_{i}\right\rangle
$$

where $\bar{x}_{i}=x_{i}+\left\langle u p^{k} x_{1}\right\rangle$ for $i=1, \ldots, n$. It can explain that simply that for $i=2, \ldots, n$ the submodule $\left\langle\bar{x}_{i}\right\rangle$ is free and $\bar{x}_{1}$ is non zero torsion element with order $o\left(\bar{x}_{1}\right)=p^{k}$. Thus $M /\langle x\rangle \cong\left\langle\bar{x}_{1}\right\rangle \oplus R^{n-1}$ with $\bar{x}_{1}$ is non zero torsion element with order $o\left(\bar{x}_{1}\right)=p^{k}$.

The following conditions apply to $R$-module $M$, u.s.dim $(M)=1$, such that in the circumstance $M=R$ is a regular module with a DVD $R$.

Lemma 3.2. Consider the DVD R. Thus u.s.dim $(R)=1$.

The requirement is that the $M$ module, which is the direct sum of free and cyclic submodules, has u.s.dim $(M)=\omega$, is as follows.
Lemma 3.3. Assume $M$ become a finitely generated $R$-module with a cyclic decomposition

$$
M=R \oplus\langle x\rangle
$$

for a torsion element $x \in M$ with its order $o(x)=p$. Hence $u . \operatorname{sim}(M)=\omega$.

Proof. We obtain the theorem if we can show that for any $N$ proper submodule of $M$ there exists $k$ such that $M / N \in \xi_{k}$ and $\forall k \in \mathbb{Z}^{+}, \exists N$ a proper submodule of $M, \ni M / N \notin \xi_{k}$. Assume $N$ become a valid submodule of $M$. When $N$ is a torsion module, hence $N \subseteq\langle x\rangle$. As long as $N \neq 0$ and $\langle x\rangle$ is simple we obtain $N=\langle x\rangle$. Hence $M / N \cong R$ a uniserial $R$-module which implies $M / N \in \xi_{1}$. The case $N$ contains a torsionfree element we can choose $y \in N, y \neq 0$ therefore $y \in R$. Thus $M /\langle y\rangle \cong R /\langle y\rangle \oplus\langle x\rangle$ is a torsion module. Moreover, $M / N$, which is isomorphic to a quotient module of $M /\langle y\rangle$, has finite uniserial dimension.

Let $k \in \mathbb{Z}^{+}$, define $N=\left\langle p^{k}\right\rangle \subseteq R \subseteq M$. Now, we obtain $M / N \cong\left(R /\left\langle p^{k}\right\rangle\right) \oplus\langle x\rangle$, a torsion module with elementary divisors $p^{k}$ and $p$. According to Theorem 2.4 state u.s.dim $(M / N)=k+1$. Thus $M / N \notin \xi_{k}$.

Lemma 3.4 below is about the uniserial dimension of a module that become a direct sum of free submodule $R$ and cyclic submodule $\oplus_{i=1}^{k}\left\langle x_{i}\right\rangle$ with order $o\left(x_{i}\right)=p^{e_{i}}$.

Lemma 3.4. Assume $M$ become a finitely generated $R$-module in line a cyclic decomposition

$$
M=R \oplus\left\langle x_{1}\right\rangle \oplus \cdots \oplus\left\langle x_{k}\right\rangle
$$

for some torsion elements $x_{i} \in M$ with order $o\left(x_{i}\right)=p^{e_{i}}$ for some positive integers

$$
e \geq e_{1} \geq \cdots \geq e_{k} \geq 1
$$

with $k \geq 1$. Hence u.s.dim $(M)=\omega+\sum_{i=1}^{k} e_{i}-1$.

Proof. The $n=\sum i=1^{k} e i-1 \geq 0$ is inserted to demonstrate lemma. For $n=0$, it implies $k=1$ and $e_{1}=1$. According to Lemma 3.3 we obtain u.s.dim $(M)=\omega=\omega+e_{1}-1$. Hence, lemma is satisfied when $n=0$.

Let $n \geq 1$ and assume lemma become valid for any $R$-module $M$ satisfying the condition in lemma with $\sum_{i=1}^{k} e_{i}-1 \leq n-1$. Suppose $M$ to be a finitely produced $R$-module. with cyclic decomposition as shown in lemma and $\sum_{i=1}^{k} e_{i}-1=n$. We will show $u . s \cdot \operatorname{dim}(M)=\omega+n$ in two steps;

- any $N$ valid submodule $M, M / N \in \xi_{\omega+n-1}$

- There is indeed a valid submodule of $M$ designated as $N$ therefore u.s.dim $(M / N)=\omega+n-1$.

Assume $N$ become a submodule, $N \neq M$. If $N$ designated as a torsion submodule, also $N \subseteq M$ is a submodule of the torsion part, that is $N \subseteq \oplus_{i=1}^{k}\left\langle x_{i}\right\rangle$. Borrowing the approach used in the proof of Theorem 2.4 there exists $x \in N, x \neq 0$ with $p x=0$ which implies

$$
M /\langle x\rangle \cong R \oplus\left(\oplus_{i=1}^{k}\left\langle y_{i}\right\rangle\right)
$$


with $o\left(y_{i}\right)=f_{i}$ and $\sum_{i=1}^{k} f_{i}=n-1$. Then, according to the induction hypothesis we obtain u.s.dim $(M /\langle x\rangle)=\omega+n-1$. Since $M / N \cong(M /\langle x\rangle) /(N\langle x\rangle)$ we obtain $M / N \in \xi_{\omega+n-1}$. For the case $N$ contains a non-torsion element, we then can show that $M / N$ is a finitely generated torsion module which implies $M / N$ has finite uniserial dimension. Hence $M / N \in$ $\xi_{\omega+n-1}$. Thus $M \in \xi_{\omega+n}$.

To show $M \notin \xi_{\omega+n-1}$, consider that $n \geq 1$ which means $\sum_{i=1}^{k} e_{i} \geq 2$. Hence $k \geq 2$ or $k=1$ with $e_{k} \geq 2$. Take $N=\left\langle p^{e_{k}-1} x_{k}\right\rangle$ then

$$
M / N \cong R \oplus\left(\oplus_{i=1}^{k}\left\langle y_{i}\right\rangle\right)
$$

with $y_{i}=x_{i}$ for $1 \leq i \leq k-1$ and $y_{k}=x+\left\langle p^{e_{k}-1} x_{k}\right\rangle$. Hence according to the induction hypothesis u.s.dim $(M / N)=\omega+$ $n-1$. Thus $M \notin \xi_{\omega+n-1}$.

From Lemma 3.4 above, we will get the following results.

Corollary 3.5. Assume $R$ become DVD, $p \in R$ is a prima element, $M$ become a finitely generrated $R$-module. Thus, u.s.dim $(M)=\omega+1$ when $M=R \oplus\langle x\rangle$ is used with $x \in M$, $\vartheta(x)=p^{2}$.

Corollary 3.6. Assume $R$ become $D V D, p \in R$ is a prima element, $M$ become a finitely generrated R-module. Hence, $u . s \cdot \operatorname{dim}(M)=\omega+k-1$ if $M=R \oplus\langle x\rangle$ with $x \in M$ and $\vartheta(x)=p^{k}$.

The uniserial dimension of free $M$ module with $r k(M)=2$ will be shown. The uniserial $M$ module is $2 \omega$, in this example.

Lemma 3.7. Consider the DVD R. Then u.s.dim $\left(R^{2}\right)=2 \omega$.

Proof.Assume $N$ be a reasonable $R^{2}$ submodule.. Then $N$ is free of rank 1 or 2 . If $\operatorname{rank}(N)=2$ then $R^{2} / N$ is torsion, finitely generated, so it has finite uniserial dimension. Thus $R^{2} / N \in \xi_{k}$ for some integer $k<2 \omega$. If $\operatorname{rank}(N)=1$ we have $N$ is a cyclic submodule of $R^{2}$. According to Lemma $3.1 M / N \cong R \oplus\langle x\rangle, \exists x \in M / N$ with $x=0$ or $x$ a torsion element. Hence, according to Lemma 3.4 can be infered $M / N \in \xi_{1}$ or $M / N \in \xi_{\omega+n}, \exists n \in \mathbb{Z}^{+}$. Hence we obtain $M \in \xi_{2 \omega}$.

To show that $\alpha=2 \omega$ is the smallest ordinal number therefore $M \in \xi_{\alpha}$ we will display that there is indeed a valid $N$ sub module $R^{2}$ for any $n$ positive integer such that $u . \operatorname{sidim}\left(R^{2} / N\right)=$ $\omega+n$ which then implies $M \notin \xi_{\omega+n}$. Let $\left\{x_{1}, x_{2}\right\}$ be a basis of $R^{2}$. Now $\forall n \in \mathbb{Z}^{+}$, take $N=\left\langle p^{n+1} x_{1}\right\rangle$. Then we have $R^{2} / N=\left\langle\bar{x}_{1}\right\rangle \oplus\left\langle\bar{x}_{2}\right\rangle$ with $\bar{x}_{i}=x_{i}+N$ for $i=1,2$. Further $\bar{x}_{1}$ is a torsion element with $o\left(\bar{x}_{1}\right)=p^{n+1}$ and $\left\langle\bar{x}_{2}\right\rangle \cong R$. Thus, according to Lemma 3.4 we obtain u.s.dim $(M / N)=\omega+n$.

The following are the main results of this section, that is the method for determining the uniserial dimension of a finitely generated free $R$-module $M \cong R^{n} \oplus\left\langle x_{1}\right\rangle \oplus \cdots \oplus\left\langle x_{k}\right\rangle$ with $n \geq 2$ and $k \geq 0$.

Lemma 3.8. Suppose $M$ become a finitely generrated $R$ module with a cyclic decomposition

$$
M \cong R^{n} \oplus\left\langle x_{1}\right\rangle \oplus \cdots \oplus\left\langle x_{k}\right\rangle
$$

for some torsion elements $x_{i}$ with $o\left(x_{i}\right)=p^{e_{i}}$ for some positive integers

$$
e \geq e_{1} \geq \cdots \geq e_{k} \geq 1
$$

with $n \geq 2$ and $k \geq 0$. Then u.s.dim $(M)=n \omega+\sum_{i=1}^{k} e_{i}$.

Proof. We will prove this lema by transfinite induction on the ordinal number $\alpha=n \omega+\sum_{i=1}^{k} e_{i}$ for non-negative integers $n \geq 2$ and $\sum_{i=1}^{k} e_{i} \geq 0$ starting from $\alpha=2 \omega$. For $\alpha=2 \omega$, we already showed that u.s.dim $\left(R^{2}\right)=2 \omega$. Thus lemma is true for $\alpha=2 \omega$.

Now let $\alpha=n \omega+m$ for some $n \geq 2$ and $m \geq 0$ and suppose for any module $M$ with a cyclic decomposition as shown in lemma with $\beta=n \omega+\sum_{i=1}^{k} e_{i}<\alpha$ implies u.s.dim $(M)=\beta$. Assume $M$ become a module of $R$ with a cyclic decomposition as shown in lemma and $\alpha=n \omega+\sum_{i=1}^{k} e_{i}$. Thus the proof can be classified into two cases; when $\alpha=n \omega$ with $n \geq 3$ and when $\alpha=n \omega+m$ with $n \geq 2$ and $m \geq 1$.

Case I: $\alpha=n \omega$. In this case $M=R^{n}$ with $n \geq 3$. We will show that $M \in \xi_{n \omega}$ and $M \notin \xi_{(n-1) \omega+m}$ for all $m$ positive integer; Thus $N$ become a valid submodule of $M$ for any positive integer of $\mu$ therefore u.s.dim $(M / N)=(n-1) \omega+\mu$.

To show $M \in \xi_{n \omega}$, Assume $N$ become a valid submodule of $R^{n}$. According to Theorem 6.7 [19, p.145] there exists $\left\{x_{1}, x_{2}, \ldots, x_{n}\right\}$ a basis of $R^{n}$ and $r_{1}, r_{2}, \ldots, r_{\ell} \in R, 1 \leq$ $\ell \leq n$ such that $\left\{r_{1} x_{1}, r_{2} x_{2}, \ldots, r_{\ell} x_{\ell}\right\}$ is a basis of $N$. Suppose $r_{i}=p^{e_{i}} u_{i}$ with $u_{i}$ units for $i=1,2, \ldots, \ell$. It is obtained that $\left\{p^{e_{1}} x_{1}, p^{e_{2}} x_{2}, \ldots, p^{e_{k}} x_{k}\right\}$ is also a basis. Thus

$$
R^{n} / N \cong\left\langle\bar{x}_{1}\right\rangle \oplus \cdots \oplus\left\langle\bar{x}_{\ell}\right\rangle \oplus R^{n-\ell}
$$

with $\bar{x}_{i}=x_{i}+N$ are torsion elements in $R^{n} / N$ with order $o\left(\bar{x}_{i}\right)=p^{e_{i}}$ for $i=1,2, \ldots, \ell$. Referring to our previous results and to the induction hypothesis we can conclude that

$$
\text { u.s.dim }\left(R^{n} / N\right)
$$

$= \begin{cases}1 & , \quad \ell=n, R^{n} / N \text { is cyclic } \\ \sum_{i=1}^{n} e_{i} & , \quad \ell=n, R^{n} / N \text { is not cyclic } \\ \omega+\sum_{i=1}^{\ell} e_{i}-1 & , \quad \ell=n-1 \\ (n-\ell) \omega+\sum_{i=1}^{\ell} e_{i} & , \quad \ell<n-1\end{cases}$

Thus u.s.dim $\left(R^{n} / N\right)<n \omega$ for any proper submodule $N$ which implies $M \in \xi_{n \omega}$. Let $\mu$ be a positive integer. To show that $M \notin \xi_{(n-1) \omega+\mu}$ let $\left\{x_{1}, \ldots, x_{n}\right\}$ be a basis of $R^{n}$ and define $N=\left\langle p^{\mu} x_{1}\right\rangle$. Then

$$
R^{n} / N=\oplus_{i=1}^{n}\left\langle\bar{x}_{i}\right\rangle
$$

where $\bar{x}_{i}=x_{i}+N$. It is easy to show that $\bar{x}_{1}$ is a torsion element in $R^{n} / N$ with order $o\left(\bar{x}_{1}\right)=p^{m}$ and for $i=2, \ldots, n$, $\left\langle\bar{x}_{i}\right\rangle$ is cyclic free submodule. Hence, according to the induction hypothesis we conclude $u . s \cdot \operatorname{dim}\left(R^{n} / N\right)=(n-1) \omega+\mu$. As a result $M \notin \xi_{(n-1) \omega+\mu}$.

Case II: $\alpha=n \omega+\sum_{i=1}^{k} e_{i}$ where $n \geq 2$ and $k \geq$ 1. In this case $M=R^{n} \oplus\left(\oplus_{i=1}^{k}\left\langle x_{i}\right\rangle\right)$ accompanied by $x_{1}, \ldots, x_{k}$ are torsion elements with order $o\left(x_{i}\right)=p^{e_{i}}$ for $i=1, \ldots, k$. We'll prove that $M \in \xi_{n \omega+\sum_{i=1}^{k} e_{i}}$ and $M \notin$ 
$\xi_{n \omega+\sum_{i=1}^{k} e_{i}-1}$; i.e. there exist a valid submodule $N$ of $M$ such that $u . \operatorname{sidim}(M / N)=n \omega+\sum_{i=1}^{n} e_{i}-1$. Suppose $N$ become a valid submodule of $R^{n} \oplus\left(\oplus_{i=1}^{k}\left\langle x_{i}\right\rangle\right)$ and $\sum_{i=1}^{k} e_{i}=l$.

1. Assume $N$ be a submodule, $N \neq M$. When $N$ designated as a torsion submodule then $N$ can be called a submodule of the torsion part of $M$, thus $N \subseteq \oplus_{i=1}^{k}\left\langle x_{i}\right\rangle$. Borrowing the approach used in the proof of Theorem 2.4, there exists $x \in N, x \neq 0$ with $p x=0$ which implies

$$
M /\langle x\rangle \cong R^{n} \oplus\left(\oplus_{i=1}^{k}\left\langle y_{i}\right\rangle\right)
$$

along $o\left(y_{i}\right)=f_{i}$ and $\sum_{i=1}^{k} f_{i}=l-1$. Then, in line with the induction hypothesis can be obtained u.s.dim $(M /\langle x\rangle)=n \omega+l-1$. Since $M / N \cong$ $(M /\langle x\rangle) /(N\langle x\rangle)$ we obtain $M / N \in \xi_{n \omega+l-1}=$ $\xi_{n \omega+\sum_{i=1}^{k} f_{i}}$.

2. For the case $N$ contains a non-torsion element $y \in$ $N$, thus can be shown that there are a non zero $y_{1} \in$ $R^{n}$ such that $y=y_{1}+\sum_{i=1}^{k} \beta_{i} x_{i}$ with $\beta_{i} \in R$, $i=1, \ldots, k$. Since $o\left(x_{i}\right)=p^{e_{i}}$ so we have $\left(\prod_{i=1}^{k} p^{e_{i}}\right) y=\left(\prod_{i=1}^{k} p^{e_{i}}\right) y_{1}$. Then there exist a non zero $z=\left(\prod_{i=1}^{k} p^{e_{i}}\right) y_{1} \in R^{n}$ therefore $\langle z\rangle$ is a submodule of $R^{n}$. Borrowing the approach used in the proof of Case I and Theorem 6.7 [19, p.145], there exist a basis $\left\{w_{1}, \ldots, w_{n}\right\}$ of $R^{n}$ and $s_{1} \in R$ therefore $\left\{s_{1} w_{1}\right\}$ is a basis of $\langle z\rangle$. Let $s_{1}=p^{f_{1}} u_{1}$ with an unit $u_{1}$. We will get that $\left\{p^{f_{1}} w_{1}\right\}$ is a basis of $\langle z\rangle$. Therefore $\langle z\rangle=\left\langle s_{1} w_{1}\right\rangle$ and we will have:

$$
M /\langle z\rangle \cong R^{n-1} \oplus\left\langle\bar{w}_{1}\right\rangle \oplus\left\langle x_{1}\right\rangle \oplus \cdots \oplus\left\langle x_{k}\right\rangle
$$

with $\bar{w}_{1}=w_{1}+\langle z\rangle$ is a torsion element of $R^{n} /\langle z\rangle$, order $o\left(\bar{w}_{1}\right)=p^{f_{1}}$ and $o\left(x_{i}\right)=p^{e_{i}}$ for $i=1, \ldots, k$. By Lemma 3.4 and hypothesis we get:

$$
\begin{gathered}
\text { u.s.dim }(M /\langle z\rangle)= \\
\left\{\begin{array}{lll}
\omega+f_{1}+\sum_{i=1}^{k} e_{i}-1 & , n-1=1 \\
(n-1) \omega+f_{1}+\sum_{i=1}^{k} e_{i} & , & n-1 \geq 2
\end{array}\right.
\end{gathered}
$$

Therefore u.s.dim $(M /\langle z\rangle)<n \omega+\sum_{i=1}^{k} e_{i}$. Since $M / N \cong(M /\langle z\rangle) /(N /\langle z\rangle)$ then can be obtain u.s.dim $(M / N)<n \omega+\sum_{i=1}^{k} e_{i}$.

From the description above, we obtain $M \in \xi_{n \omega+\sum_{i=1}^{k} e_{i}}$. To show $M \notin \xi_{n \omega+\sum_{i=1}^{k} e_{i}-1}$, take a submodule $N=\left\langle p^{e_{k}-1} x_{k}\right\rangle$ therefore:

$$
M / N \cong R^{n} \oplus\left(\oplus_{i=1}^{k}\left\langle y_{i}\right\rangle\right)
$$

with $y_{i}=x_{i}, o\left(y_{i}\right)=p^{e_{i}}$ for $1 \leq i \leq k-1$ and $y_{k}=$ $x_{k}+\left\langle p^{e_{k}-1} x_{k}\right\rangle$. By hypothesis, u.s.dim $(M / N)=n \omega+$ $\sum_{i=1}^{k} e_{i}-1$, which means $M \notin \xi_{n \omega+\sum_{i=1}^{k} e_{i}-1}$

From Lemma 3.8 above, we will get the following results.

Corollary 3.9. Think of $R$ being a $D V D, p \in R$ prima element, $M$ being a finite generrated $R$-module. Thus u.s.dim $(M)=$ $2 \omega+1$ is being utilized when $M=R \oplus R \oplus\langle x\rangle, x \in M$, $\vartheta(x)=p$.
Corollary 3.10. Think of $R$ being a DVD designated $p$ as a prima element while $M$ being a finite generrated $R$-module. Thus u.s.dim $(M)=2 \omega+k$ is being utilized when $M=$ $R \oplus R \oplus\langle x\rangle, x \in M, \vartheta(x)=p^{k}, k \geq 1$.

\section{Concluding Remarks}

In this article we proved the following results, which will close this paper.

Theorem 4.1. Assume $M$ become a finitely generated module over DVD $R$ with the preceding cyclic decomposition

$$
M \cong R^{n} \oplus_{i=1}^{k}\left\langle x_{i}\right\rangle
$$

for some torsion elements $x_{1}, \ldots, x_{k}$ with order $o\left(x_{i}\right)=$ $p^{e_{i}}, i=1, \ldots, k$ for some positive integers $e_{1} \geq \cdots \geq e_{k} \geq 1$ and non-negative integers $n, k$. Then

$$
\text { u.s.dim }(M)= \begin{cases}1 & , \quad M=R \text { or } M=\left\langle x_{1}\right\rangle \\ \sum_{i=1}^{k} e_{i} & , \quad n=0 \text { and } k \geq 2 \\ \omega+\sum_{i=1}^{k} e_{i}-1 & , \quad n=1 \text { and } k \geq 1 \\ n \omega+\sum_{i=1}^{k} e_{i} & , \quad n \geq 2 \text { and } k \geq 0\end{cases}
$$

\section{Acknowledgements}

The authors also thank referees for useful comments and suggestions which make this article better during the peer review process and reduce meaningless sentences.

\section{REFERENCES}

[1] Arifin, S., Garminia, H., "Uniserial dimension of module Zm x Zn over Z using Python,” Int. J. Sci. Technol. Res., 8, 2019, p 194-9.

[2] Arifin, S., Garminia, H., and Astuti, P., "Dimensi Valuasi Dari Daerah Ideal Utama," Prosiding Seminar Nasional Aljabar, Penerapan dan Pembelajarannya, Sanata Dharma University, 2016, p 9-16.

[3] Arifin, S., Garminia, H., and Astuti, P., "Uniserial Dimensions of Finitely Generated Primary Modules Over A Discrete Valuation Domain", In Journal of Physics: Conference Series, IOP Publishing, Vol. 1245, No. 1, 2019, p. 012057. DOI: 10.1088/1742-6596/1245/1/012057

[4] Arifin, S., Muktyas, I.B., and Sukmawati, K.I., "Product of two groups integers modulo $\mathrm{m}, \mathrm{n}$ and their factor groups using python", In Journal of Physics: Conference Series, IOP Publishing, Vol. 1778, No. 1, 2021, p. 012026. DOI: 10.1088/1742$6596 / 1778 / 1 / 012026$

[5] Atiyah, M. F. and MacDonald, I. G., "Introduction to Commutative Algebra," Addison-Wesley Publication Co., London, 1969. 
[6] Dummit, D.S., "Abstract Algebra," 3rd Edition, John Wiley and Son, Inc, 2004.

[7] Eisenbud, D. and Griffith, P., ”Serial Rings," J. Algebra, 17, 1971, p 389-400.

[8] Facchini, A. and Salce, L., "Uniserial modules: sums and isomorphisms of subquotients," Comm. Algebra 18, 2, 1990, p 499-517.

[9] Faith, C., "The structure of valuation rings," J. Pure Appl. Algebra, 31, 1984, p 7-27.

[10] Faith, C., "The structure of valuation rings II," J. Pure Appl. Algebra, 42, 1986, p 37-43.

[11] Gallian, J.A., "Contemporary Abstract Algebra," 9th Edition, USA, 2017.

[12] Ghorbani, A. and Nazemian, Z., "On commutative rings with uniserial dimension,' Journal of Algebra and Its Aplications, 14, 1, 2015, p 1-10. DOI: 10.1142/S0219498815500085

[13] Kaplansky, I., ”Modules over Dedekind rings and valuation rings," Trance. Amer. Math. Soc., 72, 1952, p 327-340.

[14] Lam, T. Y., "A First Course in Noncommutative Rings," Grad. Texts in Math., Springer, New York, 1991.

[15] Manis, M., "Valuation on a commutative ring," Proc. Amer. Math. Soc, 20, 1969, p 193-198
[16] Mostafa Ftouhi , Mohammed Barmaki, Driss Gretete, "Geometric Topics on Elementary Amenable Groups," Mathematics and Statistics, Vol. 8, No. 2, pp. 121 - 125, 2020. DOI: $10.13189 / \mathrm{ms} .2020 .080207$

[17] Nazemian, Z., Ghorbani, A. and Behboodi, M., "Uniserial dimension of modules," J. Algebra, 399, 2014, p 894-903. DOI: 10.1016/j.jalgebra.2013.09.054

[18] Puguh Wahyu Prasetyo, Indah Emilia Wijayanti, Halina France-Jackson, Joe Repka, "Weakly Special Classes of Modules," Mathematics and Statistics, Vol. 8, No. 2A, pp. 23 - 27, 2020. DOI: $10.13189 / \mathrm{ms} .2020 .081304$

[19] Roman, S., "Advanced Linear Algebra," Springer, New York, 2008.

[20] Rotman, J. J., "Advanced Modern Algebra," Prentice Hall, New York, 2003.

[21] Stoll, R. R., "Set Theory and Logic," Dover Publication Inc., New York, 1961.

[22] Warfield, R. B., "Serial rings and finitely presented modules," J. Algebra, 37, 1975, p 187-222. 